\title{
Implementation of Video Voice over IP in Local Area Network Campus Environment
}

\author{
Mohd Nazri Ismail
}

\begin{abstract}
In this research, we propose an architectural solution to integrate the video voice over IP (V2oIP) services in campus environment network. Voice over IP (VoIP) technology has become a discussion issue for this time being. Today, the deployment of this technology on an organization truly can give a great financial benefit over traditional telephony. Therefore, this study is to analyze the V2oIP and investigate the performance areas evolved with the Quality of Service (QoS) delivered by video phone system. This study focuses on quality of voice and video, packet loss, delay time and jitter through the USB Video Phone and soft phone. In this study, network management system (NMS) is used to monitor and capture the performance of V2oIP in campus environment. In addition, the most apparent of implementing soft phone and USB phone in campus environment is to define the best situation (peak and non peak hours) that can be used in operational environment. Based on the finding result, it shows that users may experience V2oIP quality service degradations due to delays and losses in the LAN during peak hours.
\end{abstract}

Key words--V2oIP, Jitter, Delay, Packet loss, CPU, Campus Environment

\section{INTRODUCTION}

As with most new technologies, Voice over Internet Protocol (VoIP) brings new challenges along with the benefits. The main challenge is VoIP's extreme sensitivity to delay and packet loss compared with other network applications such as web and e-mail services. A basic understanding of VoIP traffic and of the quality metrics provided by VoIP monitoring tools will help to keep VoIP network running smoothly. Existing hardware video phones have integrated video camera and microphone and can be used independently of any other equipment [1]. Traditionally, set top boxes are video broadcast receivers and are equipped with video/audio decoder (MPEG2) and usually based on H.263 codec for video and G.711, G.723 or G.729 codecs for voice [1]. The actual multimedia content (voice and video) is usually transmitted by means of the Real-time Transport Protocol (RTP) [8].

The objective of this study is to analyze and compare the performance of the V2oIP over USB Video Phone and soft phone. In addition, it is to develop V2oIP services environment in campus. This study focuses on quality of voice and video such as packet loss, delay time, CPU usage and jitter. Today, many people are talked and communicated face to face using USB Video Phone system. When VoIP is implemented using the public Internet, users may experience quality degradations due to dynamic delays and losses in the
LAN. Packets may be lost, either in isolation or in batches, and may experience sudden delay increases [2]. Figure 1 and Figure 2 show USB Video Phone system and soft phone application will use in real network environment for the experimental.

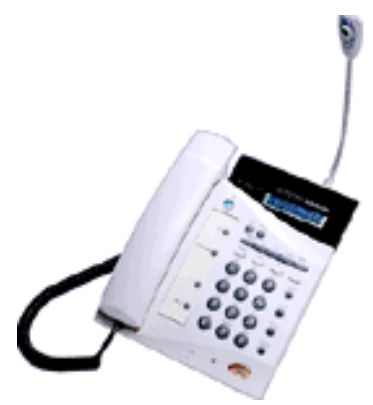

Fig. 1 USB Video Phone System

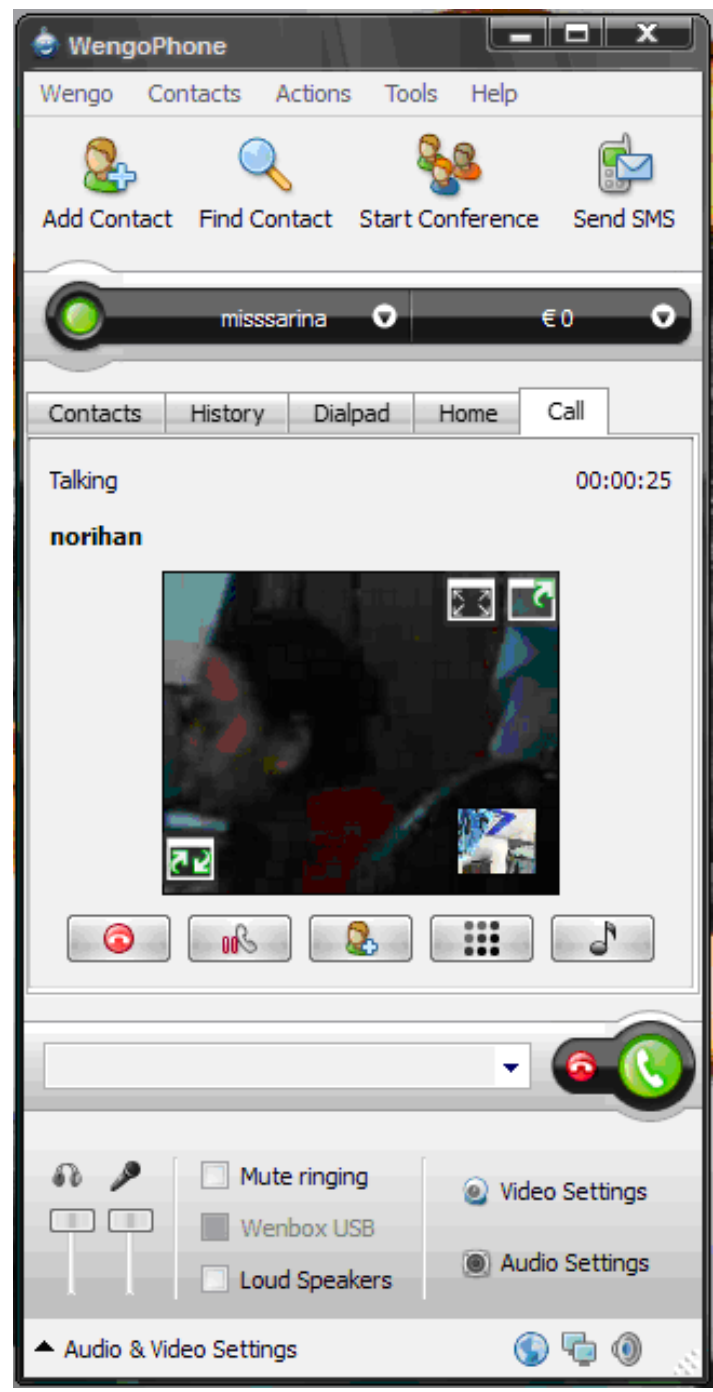

Fig. 2 Soft Phone Application 


\section{RELATED WORKS}

Recently, VoIP (Voice over IP) [3] is rapidly growing and becoming a mainstream telecommunication services, not only because of the lower cost compared with traditional PSTN (Public Switched Telephone Network), but also its convergence technologies of data and voice communication. VoIP applications like Skype [4] have also achieved great success. However, due to the complexity of the Internet, it is unpractical to calculate VoIP performance metrics only through the mathematical modeling, as what was done in the telephone networks, so the performance evaluation of VoIP requires actual measurement activities. There have been numerous studies on VoIP measurement. A. Markopoulou [5] measured loss and delay characteristics of American backbone networks, and analyzed how these characteristics impact VoIP quality. For examples, most work focused on monitoring and analyzing performance of actual applications, like MSN and Skype [6], [7], [10]. Skype using more to video codec like H.263 and H.261. Skype also using the audio codec likes G.711, G.729, G.723, G.728 and GSM.

\section{METHODOLOGY}

Figure 3 shows the overall framework of the V2oIP services in campus environment. There are five phases development process such as: i) planning and research; ii) development; iii) implementation; iv) testing and v) documentations.

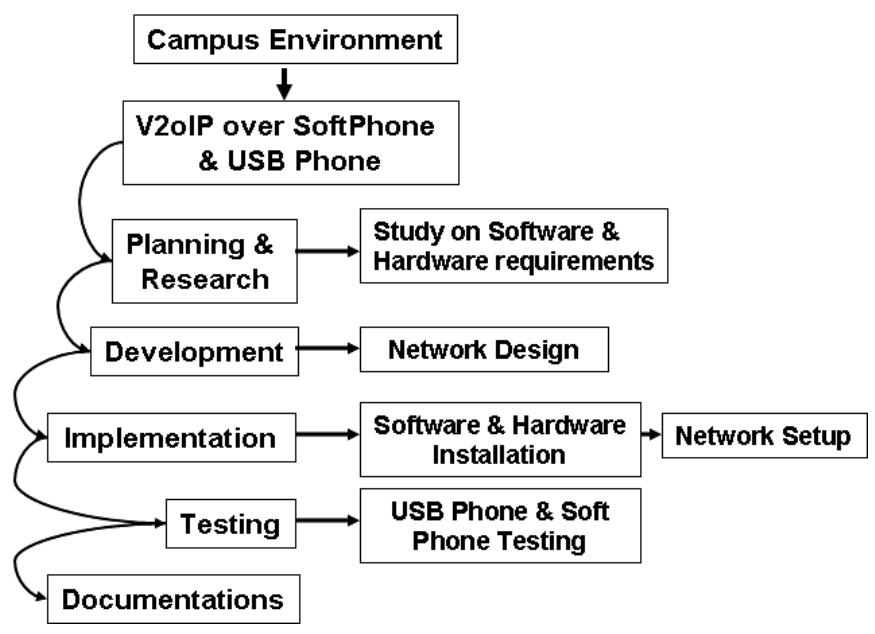

Fig. 3 Framework of V2oIP Services in Campus Environment Development

Figure 4 shows the overall framework of the V2oIP performance analysis. In the experiment, the performance analysis will focus on jitter, delay, packet loss and CPU usage over LAN. Network management system such as VQ manager and Colasoft Capsa are used to analyze V2oIP services in campus environment.

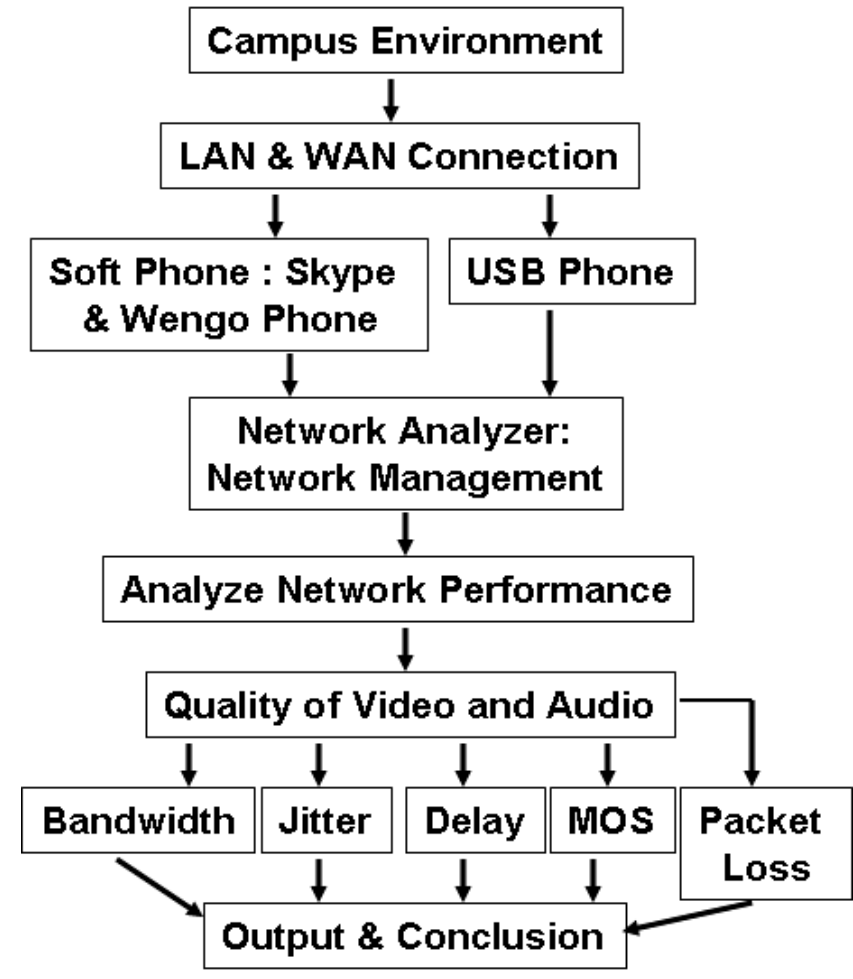

Fig. 4 Framework of V2oIP Performance Analysis

\section{PROPOSED V2OIP NETWORK ARCHITECTURE IN CAMPUS ENVIRONMENT}

We have setup a real network environment to analyze and measure implementation of V2oIP services at University of Kuala Lumpur (UniKL) in Malaysia. This study posits several research questions: i) what is the performance level of the V2oIP over LAN; and ii) Is the analysis for evaluating and measuring V2oIP performance effective?

Figure 5 shows the V2oIP architecture in real network campus environment. V2oIP quality can be monitored periodically through the measurement using VQnet (VoIP) management and colasoft capsa tools to gather quality variation information, avoiding the ignorance of unacceptable VoIP quality caused by the network failure or bandwidth bottleneck (see Figure 6).

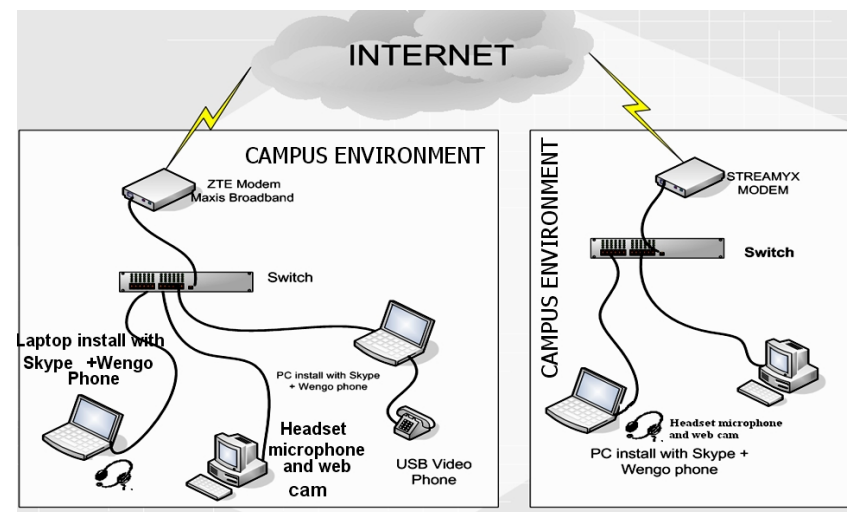

Fig. 5: Development of V2oIP Architecture in Real Network 


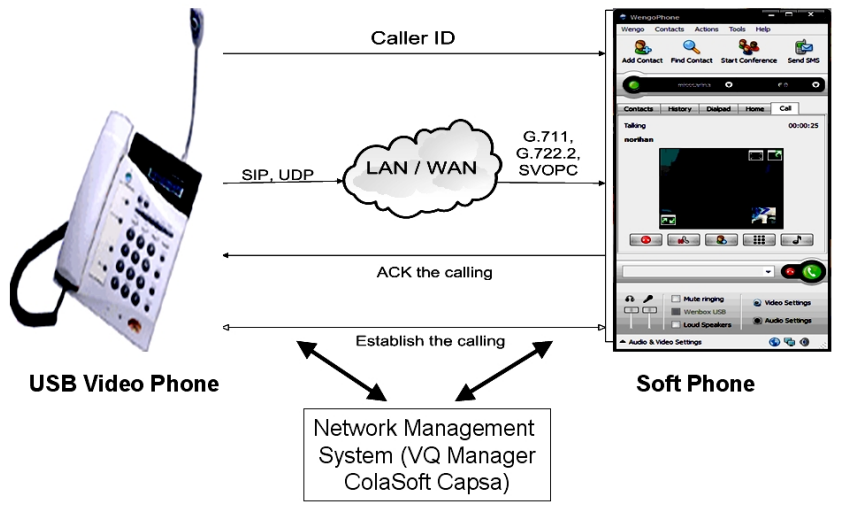

Fig. 6: Installation of Network Monitoring System for V2oIP

\section{EXPERIMENTAL AND ANALYSIS RESULTS}

This section analyzes, measures and compares V2oIP performance in campus environment. The experiment and traffic V2oIP are captured based on two situations such as: i) peak hours; and ii) non peak hours. The V2oIP traffics are measured on every two minutes. There are several VoIP software available for use in the Internet, including Skype, Google-Talk, Windows Live Messenger, Yahoo Messenger, and Gizmo Project [9]. Only, Skype can support multi-party conferencing. Result in Figure 7 and Figure 8 show that video transmission in peak hours will contribute higher delay with more network jitter that can easily occur compared to voice transmission. While, result in Figure 7 and Figure 8 show video and voice transmission in non-peak hours contribute similar and lower delay for both services (video and voice).

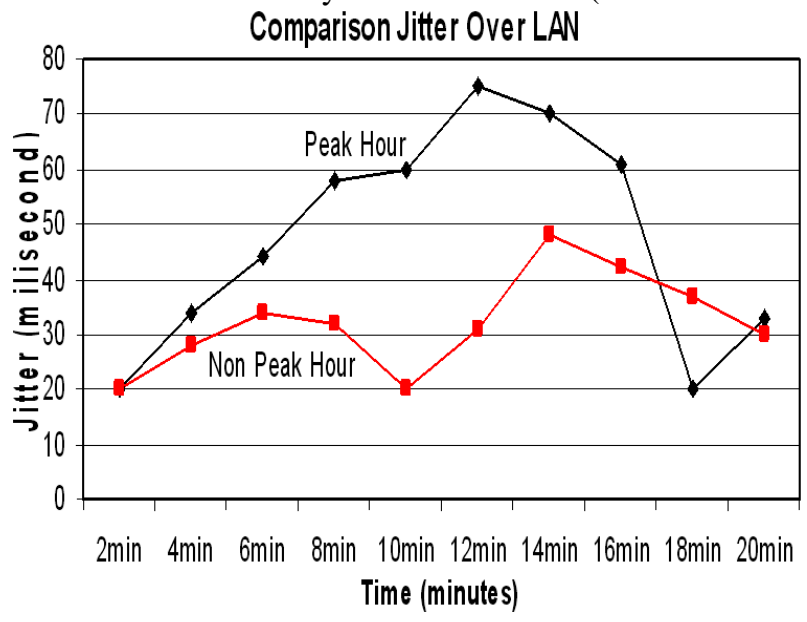

Fig. 7: Comparison of Jitter on Peak and Non Peak Hour for Voice
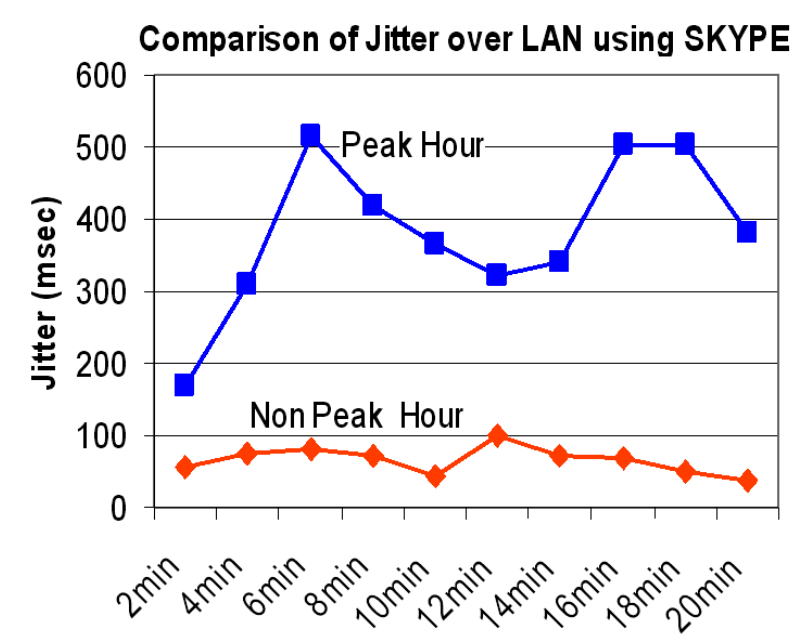

\section{Time (minute)}

Fig. 8: Comparison of Jitter on Peak and non Peak for Video

Table 1 has shown the ITU standard recommendation for delay and jitter interval which are divided in three categories as follows: i) acceptable; ii) acceptable and fair; and iii) unacceptable. Based on ITU standard measurement, Figure 9 shows that video transmission on peak hours has achieved 'unacceptable' and 'fair' level compare to non-peak hours. Video transmission on non -peak hours indicates 'acceptable' level for video conversation between two parties. For voice transmission, it has achieved 'acceptable' level on peak and non-peak hours (see Figure 7). The efficiency of V2oIP services in campus environment can quickly deteriorates when the number of users increases in peak hours.

TABLE I: ITU STANDARD RECOMMENDATION FOR DELAY AND JITTER

\begin{tabular}{|l|l|}
\hline $\begin{array}{l}\text { Range in } \\
\text { Milliseconds }\end{array}$ & Description \\
\hline $0-150$ & Acceptable for most user applications. \\
\hline $150-400$ & $\begin{array}{l}\text { Acceptable provided that administrators are aware of the } \\
\text { transmission time and it's impact on the transmission } \\
\text { quality of user applications. }\end{array}$ \\
\hline Above 400 & $\begin{array}{l}\text { Unacoeptable for general network planning purposes, } \\
\text { however, it is recognized that in some exceptional cases } \\
\text { this limit will be exceeded. }\end{array}$ \\
\hline
\end{tabular}

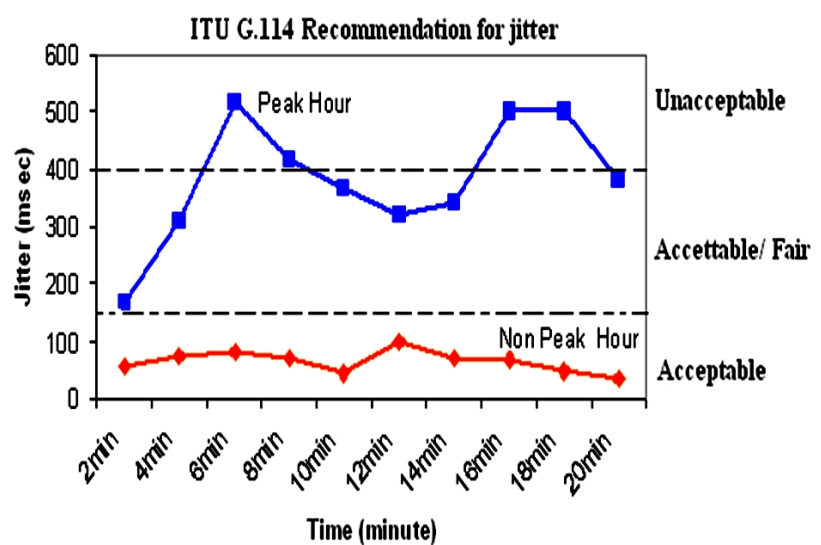

Fig. 9: Measurement of ITU Standard Recommendation for Delay and Jitter (Video Transmission)

Again, as a result in Figure 10 and Figure 11 have confirmed and proven that video transmission in peak hours 
achieve higher packet loss that can easily occur compared to voice transmission. While, result in Figure 10 and Figure 11 show video and voice transmission in non-peak hours contribute similar and lower packet loss for both services (video and voice) in campus environment.

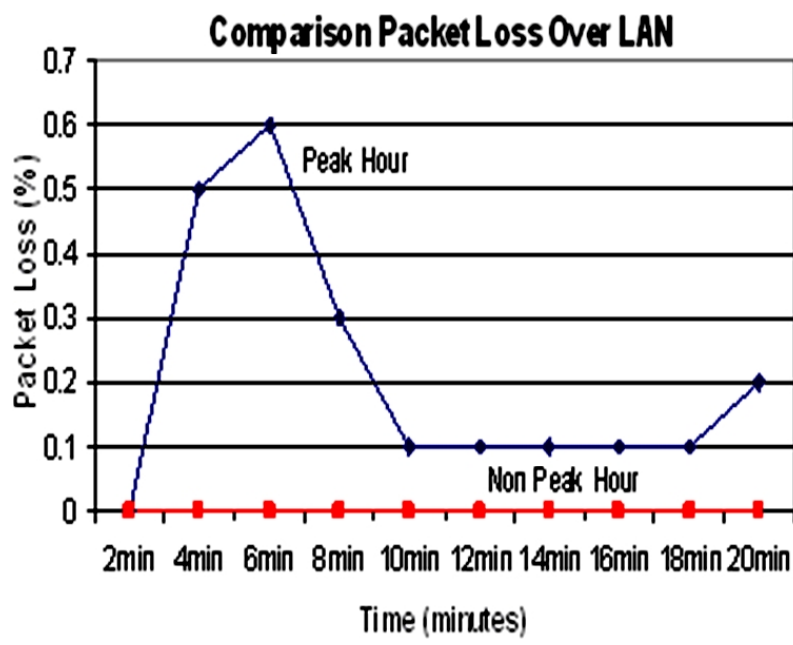

Fig. 10: Comparison of Packet Loss over LAN for Voice Services

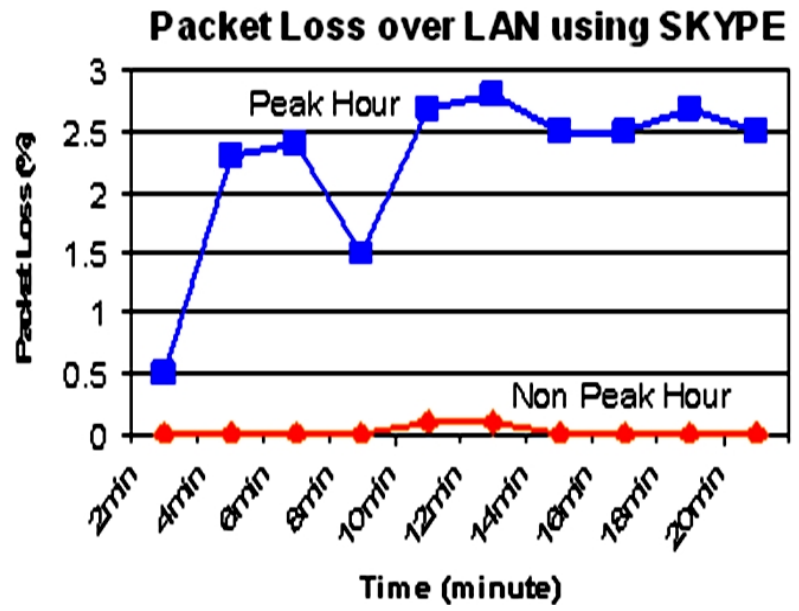

Fig. 11: Comparison of Packet Loss over LAN for Video Services

Figure 12 and Figure 13 show the CPU usage performance that affects V2oIP services especially video transmission in peak hours. Both services (video and voice) contribute different performance and characteristics. If the system load is very high, it can achieve higher CPU usage and make the throughput low that can cause inconsistent conversation between two parties. Therefore, it is important to understand how delay, jitter and CPU usage can affect speech quality. In addition, poor link quality network between two parties can also increase the delay and jitter for V2oIP services. From the analysis result, it shows that voice and video in non-peak hours have a good network performance without implementation of any QoS level in campus environment. The results of this study show that voice service is able to contribute and achieve reliability and scalability in peak and non-peak hours. Therefore, proper plan can mitigate most of the negative effects that are related to V2oIP characteristics.

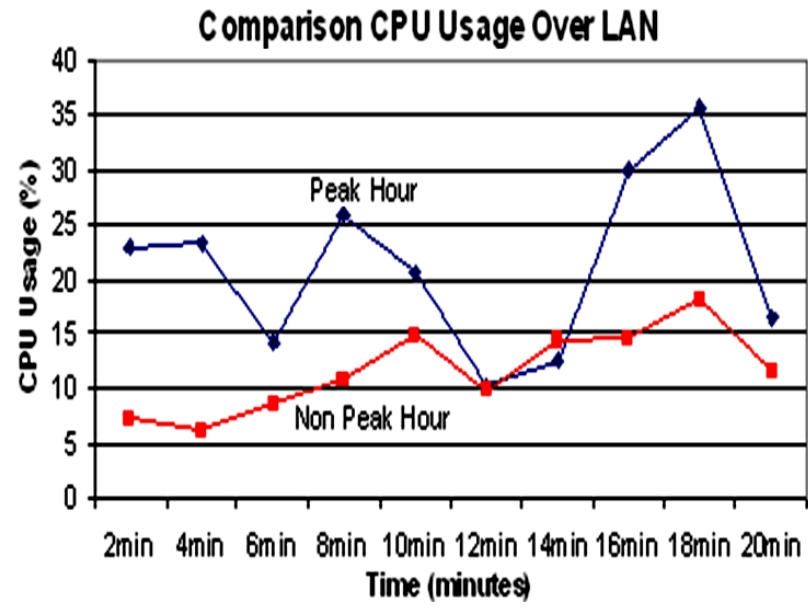

Fig. 12: Comparison of CPU Usage over LAN for Voice Services.

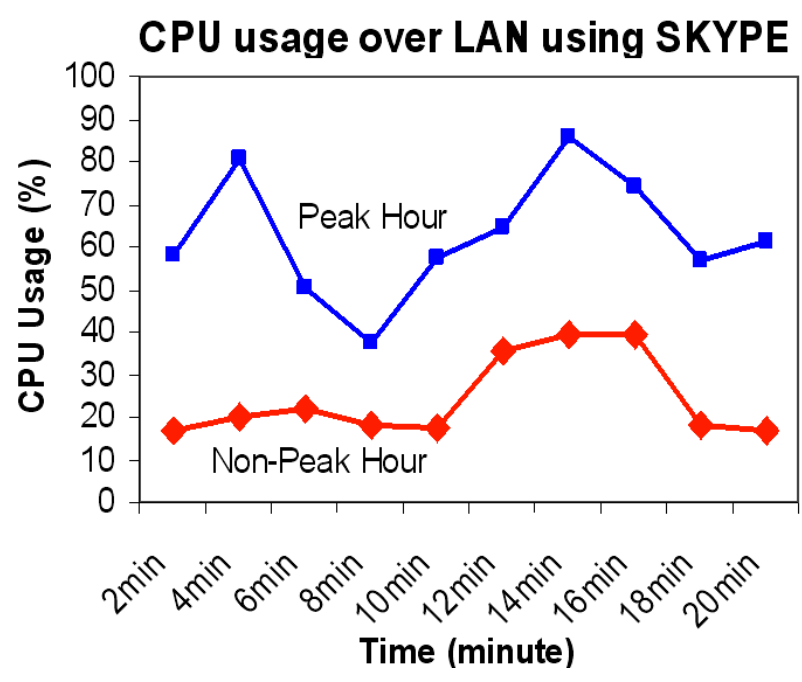

Fig. 13: Comparison of CPU Usage over LAN for Video Services

\section{CONCLUSION}

Convergence of video voice over IP with current VoIP system can contribute many revenues to VoIP users. This paper discussed which services between video and voice could produce good performance. We conclude that base on our findings, video transmission in peak hours can contribute higher delay, packet loss and CPU usage compare to voice transmission in campus environment. Non-peak hours, voice and video transmission will generate approximately similar results. If V2oIP want to implement in campus environment, it is recommended to enable QoS function in order that to achieve a good quality conversation between two parties. Mean Opinion Score (MOS) and bandwidth analysis over WAN will consider in future work. It should consider implementing techniques to improve quality of V2oIP over WAN. Therefore, efficient and effective QoS provisioning techniques are important to achieve a robust service. There are several techniques should be studied and analyzed that can be used to increase performance of V2oIP in campus environment as follows: i) dejitter buffer; ii) Type of Service (ToS); iii) Weighted Fair Queuing (WFQ); and iv) Random Early Detection (RED). Implementing quality of service mechanisms on peak hours is a method to improve V2oIP network services performance in campus environment. 


\section{REFERENCES}

[1] 'A Ne UPnP Architecture for Distributed Video Voice over IP

[2] B. Sat and B. W. Wah. Evaluation of conversational voice quality of the Skype, Google-Talk, Windows Live, and Yahoo Messenger VoIP systems, In IEEE Int'l Workshop on Multimedia Signal Processing, (accepted to appear) Oct. 2007

[3] Daniel Collins, Carrier Grade Voice over IP, 2nd Ed., McGraw-Hill, September 2002.

[4] S. A. Baset, and H. Schulzrinne, "An analysis of the Skype peer-to-peer internet telephony protocol", In Proc. of IEEE INFOCOM, Barcelona, Spain, Apr. 2006

[5] A. Markopoulou, F. Tobagi, and M. Karam, "Assessment of VoIP quality over Internet backbones", in Proc. of IEEE INFOCOM, New York, NY, Jun. 2002.

[6] K. Chen, C. Huang, P. Huang, and C. Lei, "Quantifying Skype user satisfaction", In Proc. of ACM SIGCOMM, Pisa, Italy, 2006.

[7] W. Chiang, et al., "A performance study of voip applications: Msn vs. skype", In Proc. of MULTICOMM, 2006.

[8] H. Schulzrinne, S. Casner, V. Jacobson, and R. Frederick, "RTP: A Transport Protocol for Real-Time Applications", RFC 3550, June 2003.

[9] 'The Design of a Multi-Party VoIP Conferencing System over the Internet'. Ninth IEEE International Symposium on Multimedia 2007

[10] B. Sat and B.W.Wah. "Analysis and evaluation of the Skype and Google-Talk VoIP systems”. In Proc. IEEE Int'l Conf. on Multimedia and Expo, July 2006 